Cytomechanical and topological investigation of MCF-7 cells by scanning force microscopy

This content has been downloaded from IOPscience. Please scroll down to see the full text. 2009 Nanotechnology 20055103

(http://iopscience.iop.org/0957-4484/20/5/055103)

View the table of contents for this issue, or go to the journal homepage for more

Download details:

IP Address: 35.8.11.2

This content was downloaded on 22/02/2015 at 22:42

Please note that terms and conditions apply. 


\title{
Cytomechanical and topological investigation of MCF-7 cells by scanning force microscopy
}

\author{
Stefano Leporatti ${ }^{1}$, Daniele Vergara, Antonella Zacheo, \\ Viviana Vergaro, Giuseppe Maruccio, Roberto Cingolani and \\ Ross Rinaldi
}

National Nanotechnology Laboratory (NNL) of CNR-INFM, IIT Research Unit, Scuola Superiore ISUFI, University of Salento, Via Arnesano 73100 Lecce, Italy

E-mail: stefano.leporatti@unile.it

Received 12 September 2008, in final form 20 November 2008

Published 9 January 2009

Online at stacks.iop.org/Nano/20/055103

\begin{abstract}
Despite enormous advances in breast cancer biology, there is an increased demand for new technologies/methods that are able to provide supplementary information to genomics and proteomics. Here, we exploit scanning force microscopy (SFM) in combination with confocal microscopy, to investigate the morphological and mechanical properties of two neoplastic cell lines: (i) MCF-7 (human breast cancer) and (ii) HeLa (human cervical carcinoma). Living and fixed cells either in phosphate buffer solution (PBS) or in air have been studied, and the viscoelastic properties (including the Young's modulus) of cells grown onto standard and modified (e.g. by fibronectin, one of the cellular matrix components) substrates have been measured. We observed different Young's modulus values, influenced by the adhesion and growth behaviour onto specific substrate surfaces.
\end{abstract}

\section{Introduction}

In the last decades, great attention was focused on the close relationship between cell functions and structure. In particular, cell structure is characterized by specific mechanical properties which, in turn, depend upon the cytoskeleton (a complex polymer network composed of actin filaments, microtubules and intermediate filaments (IFs)) and its organization [1]. Pioneering studies demonstrated the correlation of the mechanical properties of cells (such as cellular stiffness) with several processes including cell growth, adhesion, differentiation, locomotion, migration, invasion and cancerogenesis [2, 3], evidencing how these processes are related to cytoskeleton-dependent modifications. For example, human bronchial epithelial cells showed an increase in cytoskeleton stiffness during spreading [4]. Differences in the Young's modulus between normal and cancerous human epithelial cells were also observed, due to a different organization of the cell cytoskeleton [5]. Moreover, it was recently demonstrated that metastatic cancer cells, from

\footnotetext{
1 Author to whom any correspondence should be addressed.
}

patients with suspected lung, breast and pancreatic cancer are softer than healthy ones [6]. All these reports highlight the importance of the cytoskeleton as not merely a scaffold. So, understanding cytoskeletal perturbations can help us to comprehend and probably predict many biological processes, considering also that cytoskeletal proteins do not work in isolation but are complexed with different sets of actin-binding proteins with multiple cellular roles [7].

Nowadays, different experimental methodologies to study the mechanical properties of cells are available [8]. However, in this field, thanks to their versatility and resolution, scanning force microscopy (SFM) approaches represent unique tools to investigate almost any type of surface and biological sample in its natural aqueous environment. Various dynamic cellular processes can be followed over the course of time. Moreover, SFM allows for recording force-distance curves which by means of specific treatments can be converted into force-indentation curves whose analysis with appropriate theoretical models (such as the Hertz model and its Sneddon variation, the theory of elastic shells or finite element methods [9-11]) provides quantitative information on the nanomechanical properties of the sample. By recording 
spatially resolved force curves (or alternatively using force modulation techniques), SFM also enables the production of two-dimensional maps of the sample elasticity and thus enables a study of the spatial distribution of mechanical properties to reveal their heterogeneities. As a consequence, SFM studies can offer many details about cellular organization and dynamical rearrangements in the cytoskeleton, for example in response to drug-induced changes [12].

So far, the mechanical properties of animal cardiomyocytes, macrophages, endothelial cells, fibroblasts and osteoblasts, plant and microbial cells have been investigated [13-18]. Moreover, in the last few years, SFM studies concerning the imaging and elastic properties of neoplastic cells have also been reported [1]. Here we exploit SFM to investigate the surface morphology and mechanical properties of the MCF-7 breast cancer cell line, which represents a well-known model system used in many biomedical research laboratories for the study of breast cancer, the most common cancer among women worldwide [19]. The results are then compared with data from HeLa cells and MCF-7 cultured on fibronectin (with respect to an unmodified plastic substrate). To our knowledge, this is the first systematic nanomechanical study of MCF-7 cells, an important step for a deeper understanding and analysis of breast cancer.

\section{Materials and methods}

\subsection{Cell culture and fixation}

MCF-7 and HeLa cells were seeded in $25 \mathrm{~cm}^{2}$ flasks at a concentration of $1 \times 10^{4} \mathrm{~cm}^{-2}$ using DMEM medium (Sigma) supplemented with 10\% FCS (Sigma) and antibiotics (100 units $\mathrm{ml}^{-1}$ penicillin and $100 \mu \mathrm{g} \mathrm{ml}^{-1}$ streptomycin). The cells were cultured at $37^{\circ} \mathrm{C}$ with $5 \% \mathrm{CO}_{2}$.

Immediately before performing the SFM analysis, the cells were washed with phosphate-buffered saline (PBS) solution and the medium solution was replaced with $2 \mathrm{ml}$ of Leibovitz's L-15 medium (Sigma). Cell fixation was carried out for 5 min with $0.25 \%$ glutaraldehyde in PBS. After extensive washing, the cells were resuspended in PBS or dehydrated by addition of increasing concentrations of ethanol, and were air dried.

Coating of $35 \mathrm{~mm}$ plastic Petri dishes (Corning) with fibronectin (Sigma) was performed by adding the protein at $10 \mu \mathrm{g} \mathrm{cm}^{-2}$ in PBS at room temperature for $1 \mathrm{~h}$. After washing, the dishes were immediately used for experiments.

\subsection{Confocal microscopy}

For confocal studies, the cells were fixed in 3.7\% formaldehyde in PBS for $5 \mathrm{~min}$, and permeabilized with Triton X-100 $(0.1 \%$ in $\mathrm{PBS})$, followed by a $30 \mathrm{~min}$ incubation at room temperature with phalloidin-TRITC (Sigma). The cells were then washed with PBS solution for $5 \mathrm{~min}$ and subsequently stained with $1 \mu \mathrm{g} \mathrm{ml}^{-1}$ of Hoechst 33342 for $5 \mathrm{~min}$ at room temperature. Images were acquired by confocal microscopy using a TCS SP5 microscope (Leica Microsystem GmbH, Mannheim, Germany).

\subsection{Scanning force microscopy (SFM)}

SFM imaging and force mapping was conducted using a Bioscope 2 system (Veeco Instruments Inc., Santa Barbara, CA, USA) mounted on an inverted optical microscope (Zeiss Observer Z1, Carl Zeiss, Goettingen, Germany) which enables direct optical access to the sample (located in a Petri dish) and to position the SFM's tip on a selected cell or cell portion (with a lateral resolution around $1 \mu \mathrm{m}$ ). Living cells were imaged in Leibovitz culture medium (L15) for a maximum of two hours by using a heating device to maintain the temperature of the Petri dish constant $\left(37^{\circ} \mathrm{C}\right)$, whereas glutaraldehydefixed cells were imaged both in contact and tapping modes in air or in PBS. For contact mode imaging, in order to avoid cell damaging we used V-shaped silicon nitride cantilevers with very low constants, ranging from 0.01 to $0.05 \mathrm{~N} \mathrm{~m}^{-1}$ (Microlever, MLCT-AUNM, Veeco, USA). For tapping mode imaging we used RTESPA (Veeco, USA) silicon cantilevers of about $40 \mathrm{~N} \mathrm{~m}^{-1}$ spring constant and resonance frequency of around $300 \mathrm{kHz}$.

In constant force mode a feedback loop controls the piezo's Z-height to keep (as much as possible) the cantilever deflection constant, and, simultaneously with the topography, it is possible to obtain a deflection image which can be roughly considered as a directional derivative of the sample topography. Concerning force spectroscopy, the loading force is proportional to the deflection via the cantilever's spring constant, and we calibrated the sensitivity of the optical system and determined the cantilever spring constant before each experiment using built-in routines from the manufacturer. Specifically, the optical lever sensitivity (necessary to convert the voltage applied to the piezo into deflection distance of the cantilever in nanometres) was determined on a bare region of the Petri dish from force versus distance curves. Then, the cantilever spring constant was measured using the thermal noise method $[9,10]$.

To measure the elastic properties quantitatively, we recorded force curves measuring the tip deflection as a function of the sample height while the tip was moved towards the sample. From these curves we obtained the elastic indentation as a function of the loading force applied by the tip. Since the Hertz model describes just a simple case of elastic deformation of two perfectly homogeneous smooth bodies touching under load, the local Young's modulus $[11,21]$ was evaluated by fitting the experimental data with the Sneddon variation of the Hertz model, which is more appropriate and assumes a simplified stiff-cone geometry for the SFM tip indenting a soft flat surface (the cell in our case). For mapping the elastic properties, we operated in the so-called force mapping mode (force volume) which allows us to record two-dimensional arrays of force curves while the SFM's tip is raster-scanned across the area under investigation. A trigger algorithm ensures that the maximum loading force does not exceed a preselected value (usually from a few $\mathrm{nN}$ to $20-30 \mathrm{nN}$ ) defined in order to avoid excessive cell indentation [20]. Single approach curves were analysed using a Matlab home-written routine. At least 15 different cells were probed for each condition and at least 10 different force curves on the whole area of each cell (comprising the nucleus) were analysed. Since we observed 

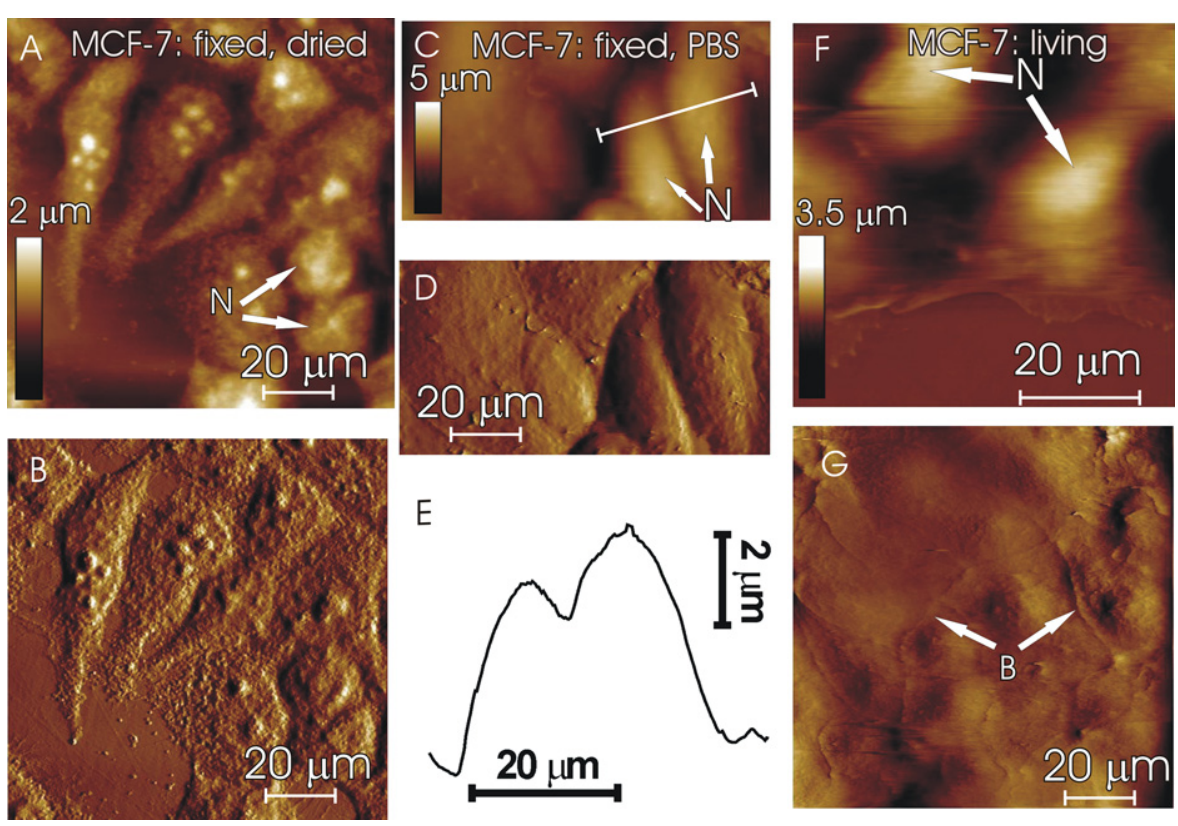

Figure 1. SFM images of MCF-7 cells. ((A), (B)) Height and corresponding deflection images of fixed and dried cells; ((C)-(E)) height, deflection image and Z-profile of cells in buffered solution. $((\mathrm{F}),(\mathrm{G}))$ Height and (not corresponding) deflection images of living cells in L-15 cultured medium from a cell colony and its border. Arrows indicate nuclei $(\mathrm{N})$ which appear as bright features in height mode and cell borders (B).

(This figure is in colour only in the electronic version)

changes in the elastic modulus as a function of indentation depth, we compared the values (averaged for each single cell) corresponding to an indentation range between 200 and $600 \mathrm{~nm}$. The distributions of Young's modulus values for different cells and experimental conditions were statistically analysed and compared by using a two-sample independent $t$-test. Differences between force curves were considered significant at $P<0.05$.

\section{Results and discussion}

\subsection{SFM and confocal imaging}

MCF-7 cells have a typical epithelial-like morphology in the culture flask/Petri dish. However, the cells seeded on the surface of the plastic Petri dish have the tendency to form dense colonies, resulting in difficulties for SFM imaging at the cellular level and in probing lateral domains involved in cell-cell interactions. For this reason, before performing the SFM study, the plastic Petri dish was examined microscopically to choose an area containing isolated groups of few cells. Deflection images are reported together with corresponding topography images since small corrugations and morphological details are more clearly visible there.

SFM images of fixed and dried cells (figures 1(A) and (B)) allowed us to obtain much detail about the shape of the cells and their interactions with the substrate. Images were obtained in both contact mode and tapping mode (data not shown); no differences between cell images were observed in terms of surface structure resolution. The cells appear quite flat (a result of the drying procedure) with a prominent nucleus in the centre surrounded by its nuclear envelope. As is well known, the nucleus is a dynamic organelle [22], characterized by a specific subnuclear organization with a unique set of organelles [23]. Among these, different nucleoli are easily visible in our SFM images in the nuclear region (see the round-shaped protrusions in figures $1(\mathrm{~A})$ and $(\mathrm{B})$ ).

For comparison, we report SFM images of fixed cells in PBS (figures 1(C), (D), while figures 1(A) and (B) correspond to air-dried cells) and living cells in L-15 medium (figures $1(\mathrm{~F}),(\mathrm{G})$ ). The macroscopic shape of living cells is similar to that observed for fixed cells in PBS: in both images central nuclei $(\mathrm{N})$ and cell borders (B) can be easily identified (see arrows). However, the cell membrane architecture appears different after the fixation step. In fact, living cells showed fine details of the cell membrane (figure $1(G)$ ), which were not so evident on the surface of fixed cells imaged in PBS (figures 1(C), (D)).

To obtain information about the organization of actin filaments, SFM observations were complemented with confocal observations. This is an important point since the role of the filaments in the regulation of cellular processes linked to proliferation, apoptosis and invasion [24, 25] and their contribution to the cell stiffness [26] is widely accepted. For this purpose, MCF-7 cells were fluorescently labelled using TRITC-phalloidin and Hoechst to stain the F-actin and nuclei, respectively. A typical confocal image is shown in figure 2: actin filaments are distributed mainly around the cell periphery without the presence of other structural elements, for example stress fibres, in the cytoplasmatic region. It should be noted that, similarly to what was observed by confocal microscopy, in SFM images cell borders were also clearly shown, and a negligible number of actin stress fibres were observed in the 


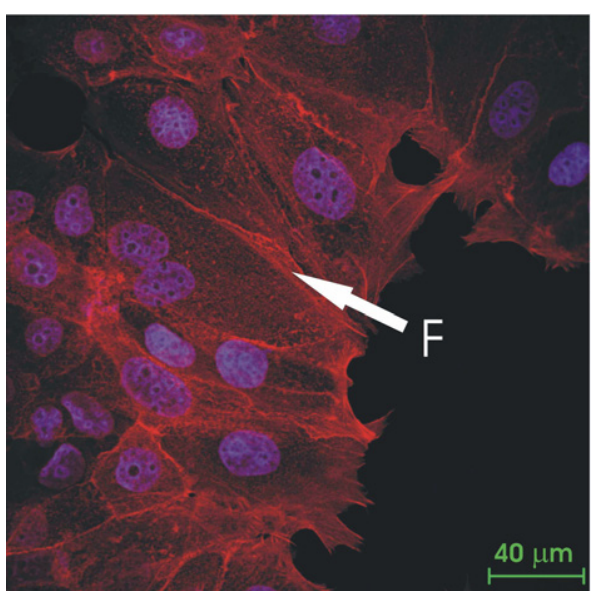

Figure 2. Confocal image of F-actin staining with TRITC-phalloidin in MCF-7 cells. The nuclei were counterstained with Hoechst (blue). The arrow indicates F-actin filaments $(\mathrm{F})$.

central portion of the cell. Moreover, figure 2 also shows how direct interactions between cells (which cross-link one cell with another) as well as between cells and the substrate are mediated by several cytoplasmatic projections that contain actin filaments (F, indicated by the arrow).

\subsection{Determination of Young's modulus in fixed and living cells}

Few direct comparison of the mechanical properties of human cancer cells have been made $[5,6]$. In order to investigate how structural differences in the organization of the cytoskeleton affect the elastic properties of cancer cells, a comparison was done between MCF-7 and the cervical cancer cell line HeLa. In fact, these two cell lines exhibit similar phenotypic characteristics but a different cytoskeletal structure. The results obtained by comparing force-indentation curves are summarized in table 1 . In both cell lines, particularly striking is the great difference between fixed and living cells in terms of elasticity and stiffness, a result of fixation in glutaraldehyde as well reported in the literature [27].

The results obtained from HeLa cells are comparable to those of Gigler and collaborators [28]. In fact, those authors reported an elasticity Young's modulus value in a range of 50$500 \mathrm{kPa}$ for living cells, which is comparable to our results.

Differences in cell elasticity measurement were observed between the two cell lines: MCF-7 cells were more than two times softer than HeLa cells in the same experimental conditions (figure 3). We believe that the different organization of the cytoskeleton could be responsible for these great differences; in particular, intermediate filaments (IFs, not easily identifiable without the use of a specific fluorescent marker) could play a pivotal role. IFs are a heterogeneous group of proteins having a primarily structural role inside the cells that have been divided into five groups with a celltype specific expression [29]. IFs together with the actin filaments have been identified as the main determinants of cell viscoelasticity [30].

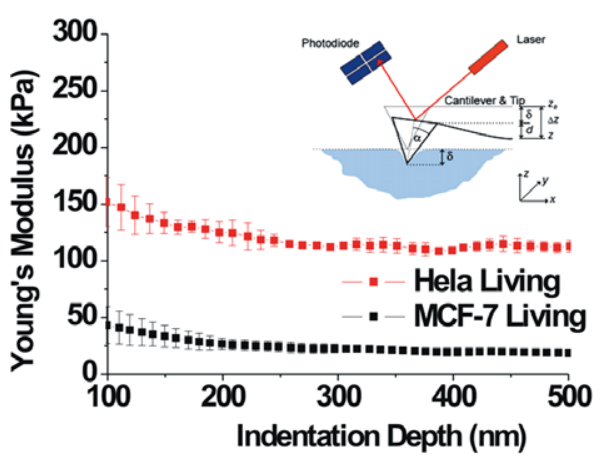

Figure 3. Characteristic Young's modulus of HeLa cells and MCF-7 living cells grown on a Petri dish as a function of indentation depth. Inset: schematic representation of SFM tip indentation on a cell.

Table 1. Ranges of Young's moduli measured for living and fixed (imaged in PBS) MCF-7 and HeLa cells.

\begin{tabular}{lll}
\hline Cell line & $\begin{array}{l}\text { Young's modulus } \\
(\mathrm{kPa})\end{array}$ & Analysed cells \\
\hline HeLa & & \\
$\quad$ HeLa Living & $100-200$ & 15 \\
HeLa Fixed & $400-500$ & 15 \\
MCF-7 & & \\
MCF-7 Living & $20-30$ & 35 \\
MCF-7 Fixed & $50-150$ & 15 \\
MCF-7 Living Fn & $15-25$ & 24 \\
\hline
\end{tabular}

The mechanical role of IFs and the contribution of IF components to cell stiffness have been established using several cell lines including fibroblasts and endothelial cells [31,32]. Magnetic twisting measurements were used to demonstrate that wild-type cells were stiffer than the vimentindeficient cells or that after vimentin IFs were disrupted by acrylamide [31].

MCF-7 and HeLa differ in their expression pattern of IFs: MCF-7 cells express keratins 7,8 and 18 but neither vimentin nor desmin; HeLa cells express both vimentin and keratins 7, 8, 17 and 18 but no desmin [33]. Even if it is difficult to extract data about the contribution arising from the mechanical properties of single filaments, the lack of expression of vimentin and the different expression of keratin isoforms could account for the different stiffness of cells.

\subsection{MCF-7 cell interactions with fibronectin}

The cellular microenvironment has a large influence on the cellular behaviour [34]. In this context, the extracellular matrix (ECM), in addition to its dual role in tissue maintenance and as a physical barrier for cancer invasion, transmits signal on target cells by specific transmembrane receptors of the integrin family [35]. Integrins function as mechanoreceptors by providing a link between the ECM and the cytoskeleton and by modulating the activation of several genes and proteins [36, 37].

Fibronectin (Fn), in particular, is an ECM glycoprotein involved in cell attachment and adhesion that mediates the interaction between the cell and other ECM proteins [38]. 

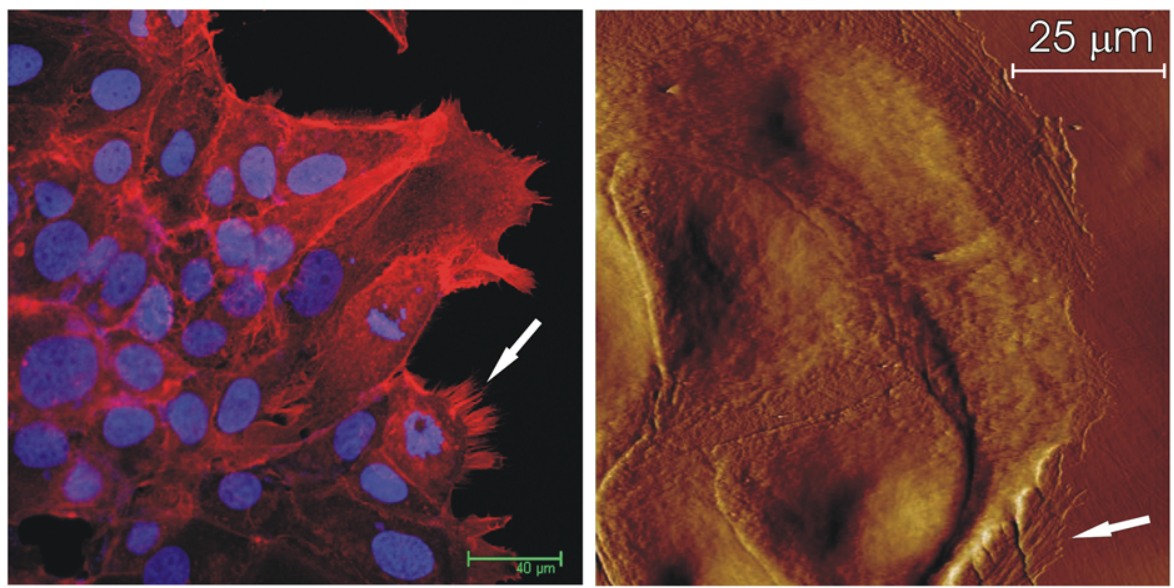

Figure 4. Laser scanning confocal microscopy (left) and SFM image (right) of MCF-7 cells grown on Fn modified Petri dish. Cells were stained with TRITC-labelled phalloidin to reveal F-actin and with Hoechst to detect nuclei. When grown on Fn, the cells exhibited short actin-rich extensions around their perimeters, as indicated by arrows, as well as cytoplasmic extensions at the cell edges. Similar features were not observed in MCF-7 grown on the Petri dish.

Fn attachment is a result of specific molecular interactions involving its $\alpha_{5} \beta_{1}$ integrin receptor.

We cultured MCF-7 cells on a fibronectin-coated substrate and analysed the morphological and elastic properties of the cells by SFM. Not surprisingly, the interaction of these with the ECM components led to modification of the cellular shape and stiffness, that were monitored and revealed by SFM. MCF-7 showed increased spreading in the presence of Fn; fine protrusions (microspikes) at the cell surface have also been noted microscopically and identified as actin-containing extensions by phalloidin staining (figure 4). Moreover, after the growth on Fn, the Young' modulus of MCF-7 was slightly lower than that found for cells grown on a plastic Petri dish (table 1).

\section{Conclusions}

Several approaches have tried to provide a contribution to the knowledge of breast cancer using the well-known MCF7 cell lines as a model. Recent techniques including proteomics [39] and microarrays [40] have also been used. With the rapid explosion of SFM technology in the biological and medical fields, decoding the biological information in terms of mechanical properties is important for obtaining the 'biomechanical signature' of tissue and cells in normal and pathological conditions.

Here, we used scanning force microscopy to obtain information about the elastic properties of MCF-7. A comparison with HeLa cells was also carried out. In addition, we noted how the interactions with surrounding Fn induce changes in the biomechanical properties. Signals from the microenvironment alter the viscoelastic properties in a manner that can or cannot facilitate tumour migration. Understanding how these modifications affect breast cancer behaviour in vivo is a great challenge and a primary goal in cancer research, considering that the process of cancer metastasis is driven by the local invasion of host stroma. In this context, cytoskeletal proteins are the main determinants of cell elasticity and a possible target for therapy. In this respect, the SFM technique is suitable for fundamental studies of the interaction of cells with the surrounding extracellular matrix.

\section{Acknowledgments}

Financial support from the Italian Ministry of University and Research (MIUR) through FIRB project no. RBLA03ER38 is gratefully acknowledged. 'Con il contributo del Ministero degli Affari Esteri, Direzione Generale per la promozione e la Cooperazione Sociale' (Italy-USA Bilateral Project 'Nanocarriers for Cancer Therapy').

\section{References}

[1] Suresh S 2007 Biomechanics and biophysics of cancer cells Acta Biomater. 3 413-38

[2] Kuznetsov Y G, Malkin A J and McPherson A 1997 Atomic force microscopy studies of living cells: visualization of motility, division, aggregation, transformation, and apoptosis J. Struct. Biol. 120 180-91

[3] Oberleithner H, Giebisch G and Geibel J 1993 Imaging the lamellipodium of migrating epithelial cells in vivo by atomic force microscopy Pfluegers Arch. 425 506-10

[4] Doornaert B, Leblond V, Planus E, Galiacy S, Laurent V M, Gras G, Isabey D and Lafuma C 2003 Time course of actin cytoskeleton stiffness and matrix adhesion molecules in human bronchial epithelial cell cultures Exp. Cell. Res. 287 199-208

[5] Lekka M, Laidler P, Gil D, Lekki J, Stachura Z and Hrynkiewicz A Z 1999 Elasticity of normal and cancerous human bladder cells studied scanning force microscopy Eur. Biophys. J. 28 312-6

[6] Cross S E, Jin Y S, Rao J and Gimzewski J K 2007 Nanomechanical analysis of cells from cancer patients Nat. Nanotechnol. 2 780-3

[7] dos Remedios C G, Chhabra D, Kekic M, Dedova I V, Tsubakihara M, Berry D A and Nosworthy N J 2003 Actin binding proteins: regulation of cytoskeletal microfilaments Physiol. Rev. 83 433-73

[8] Bao G and Suresh S 2003 Cell and molecular mechanics of biological materials Nat. Mater. 2 715-25 
[9] Hertz H 1881 Über die Berührung fester elastischer Körper J. Reine Angew. Math. 92 156-71

[10] Johnson K L 1985 Contact Mechanics (Cambridge: Cambridge University Press)

[11] Sneddon I N 1965 The relation between load and penetration in the axisymmetric Boussinesq problem for a punch of arbitrary profile Int. J. Eng. Sci. 3 47-57

[12] Rotsch C and Radmacher M 2000 Drug-induced changes of cytoskeletal structure and mechanics in fibroblasts: an atomic force microscopy study Biophys. J. 78 520-35

[13] Kliche K, Kuhn M, Hillebrand U, Ludwig Y, Stock C and Oberleithner H 2006 Direct aldosterone action on mouse cardiomyocytes detected with atomic force microscopy Cell. Physiol. Biochem. 18 265-74

[14] Leporatti S, Gerth A, Köhler G, Kohlstrunk B, Hauschildt S and Donath E 2006 Elasticity and adhesion of resting and lipopolysaccharide-stimulated macrophages FEBS Lett. $\mathbf{5 8 0} 450-4$

[15] Sato M, Suzuki K, Ueki Y and Ohashi T 2007 Microelastic mapping of living endothelial cells exposed to shear stress in relation to three-dimensional distribution of actin filaments Acta Biomater. 3 311-9

[16] Haga H, Sasaki S, Kawabata K, Ito E, Ushiki T and Sambongi T 2000 Elasticity mapping of living fibroblasts by AFM and immunofluorescence observation of the cytoskeleton Ultramicroscopy 82 253-8

[17] Grant C, Twigg P, Bell G and Lu J R 2008 AFM relative stiffness measurement of the plasticising effect of a non-ionic surfactant on plant leaf wax J. Colloid Interface Sci. 321 360-4

[18] Touhami A, Nysten B and Dufrêne Y F 2003 Nanoscale mapping of the elasticity of microbial cells by atomic force microscopy Langmuir 19 4539-43

[19] Parkin D M, Bray F, Ferlay J and Pisani P 2005 Global cancer statistics 2002 Cancer J. Clin. 55 74-108

[20] Mahaffy R E, Shih C K, MacKintosh F C and Käs J 2000 Scanning probe-based frequency-dependent microrheology of polymer gels and biological cells Phys. Rev. Lett. $85880-3$

[21] Laurent V M, Kasas S, Yersin A, Schäffer T E, Catsicas S, Dietler G, Verkhovsky A B and Meister J J 2005 Gradient of rigidity in the lamellipodia of migrating cells revealed by atomic force microscopy Biophys. J. 89 667-75

[22] Monneron A and Bernhard W 1969 Fine structural organization of the interphase nucleus in some mammalian cells J. Ultrastruct. Res. 27 266-88

[23] Handwerger K E and Gall J G 2006 Subnuclear organelles: new insights into form and function Trends Cell Biol. 16 19-26

[24] Ndozangue-Touriguine O, Hamelin J and Bréard J 2008 Cytoskeleton and apoptosis Biochem. Pharmacol. 76 11-8

[25] Friedl P and Wolf K 2003 Tumour-cell invasion and migration: diversity and escape mechanisms Nat. Rev. Cancer $3362-74$
[26] Hofmann U G, Rotsch C, Parak W J and Radmacher M 1997 Investigating the cytoskeleton of chicken cardiocytes with the atomic force microscope J. Struct. Biol. 119 84-91

[27] Hutter J L, Chen J, Wan W K, Uniyal S, Leabu M and Chan B M 2005 Atomic force microscopy investigation of the dependence of cellular elastic moduli on glutaraldehyde fixation J. Microsc. $21961-8$

[28] Gigler A, Holzwarth M and Marti O 2007 Local nanomechanical properties of HeLa-cell surfaces J. Phys. Conf. Ser. 61 780-4

[29] Hesse M, Magin T M and Weber K 2001 Genes for intermediate filament proteins and the draft sequence of the human genome: novel keratin genes and a surprisingly high number of pseudogenes related to keratin genes 8 and 18 J. Cell Sci. 114 2569-75

[30] Bausch A R and Kroy K 2006 A bottom-up approach to cell mechanics Nat. Phys. 2 231-8

[31] Wang N and Stamenovic S 2000 Contribution of intermediate filaments to cell stiffness, stiffening, and growth $A m . J$. Physiol. Cell. Physiol. 279 188-94

[32] Wang N 1998 Mechanical interactions among cytoskeletal filaments Hypertension 32 162-5

[33] Moll R, Franke W W, Schiller D L, Geiger B and Krepler R 1982 The catalog of human cytokeratins: patterns of expression in normal epithelia, tumors and cultured cells Cell 31 11-4

[34] Leporatti S, Zacheo A, Vergara D, Vergaro V, Maruccio G, Lvov Y M, Cingolani R and Rinaldi R 2008 Engineering microenvironment by layer-by-layer biocomposite films for breast cancer cells controlled growth: Morphostructure and cytomechanics study Meeting Polymer Materials Science and Engineering Proc. 236th American Chemical Society

[35] Giancotti F G 2000 Complexity and specificity of integrin signalling Nat. Cell. Biol. 2 E13-4

[36] Xie J W and Haslam S Z 2007 Extracellular matrix, Rac1 signaling, and estrogen-induced proliferation in MCF-7 breast cancer cells Breast Cancer Res. Treat. 110 257-68

[37] Fontana S, Pucci-Minafra I, Becchi M, Freyria A M and Minafra S 2004 Effect of collagen substrates on proteomic modulation of breast cancer cells Proteomics 4 849-60

[38] De Jong K L, MacLeod H C, Norton P R and Petersen N O 2006 Fibronectin organization under and near cells Eur. Biophys. J. 35 695-708

[39] Canelle L, Bousquet J, Pionneau C, Hardouin J, Choquet-Kastylevsky G, Joubert-Caron R and Caron M 2006 A proteomic approach to investigate potential biomarkers directed against membrane-associated breast cancer proteins Electrophoresis 27 1609-16

[40] Wu M, Soler D R, Abba M C, Nunez M I, Baer R, Hatzis C, Llombart-Cussac A, Llombart-Bosch A and Aldaz C M 2007 CtIP silencing as a novel mechanism of tamoxifen resistance in breast cancer Mol. Cancer Res. 5 1285-95 